\title{
Rectal Tumor to Anal Verge Distance
}

National Cancer Institute

\section{Source}

National Cancer Institute. Rectal Tumor to Anal Verge Distance. NCI Thesaurus. Code C139184.

A measurement of the distance between the rectal tumor and the anal margin. 